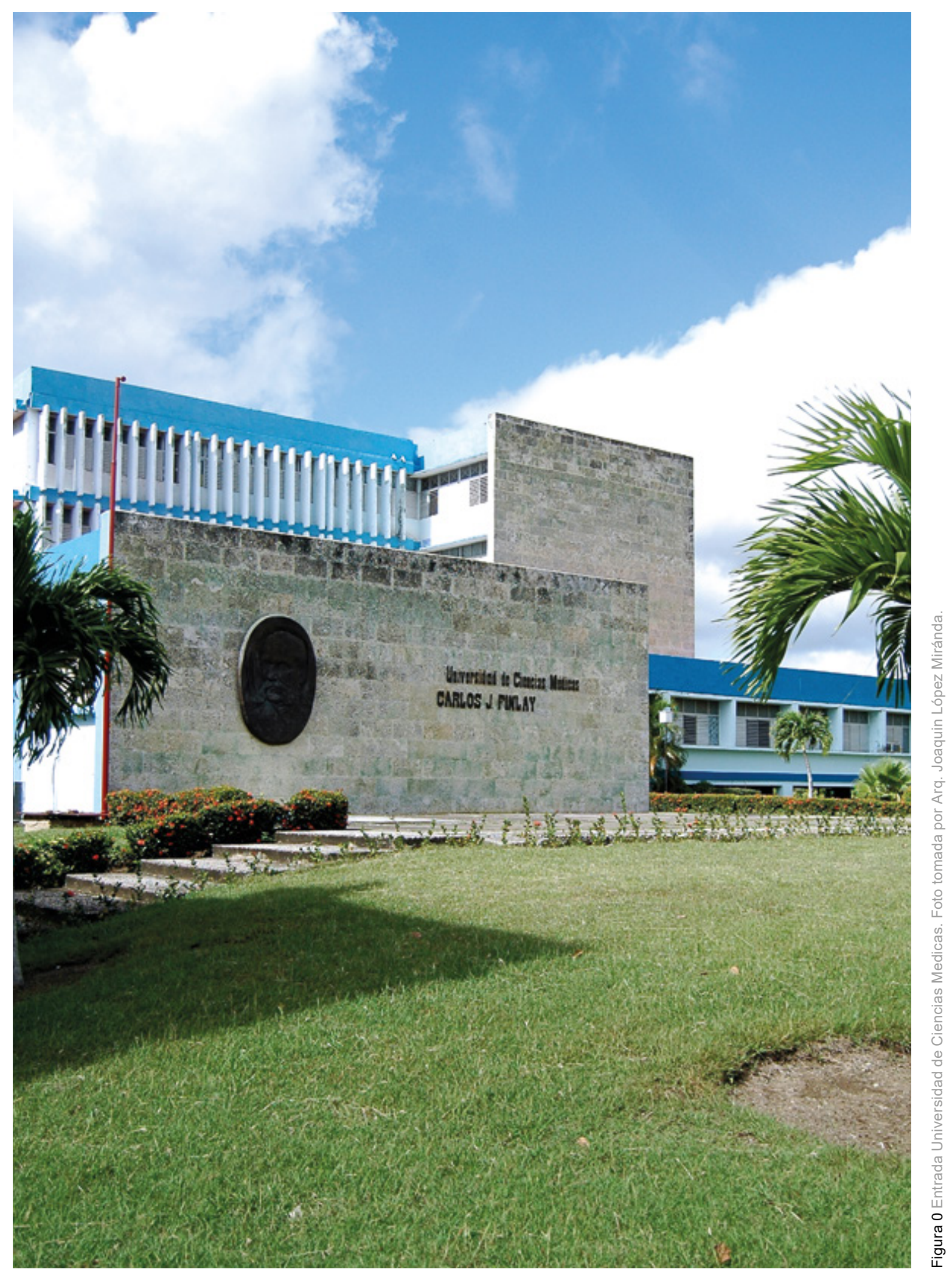



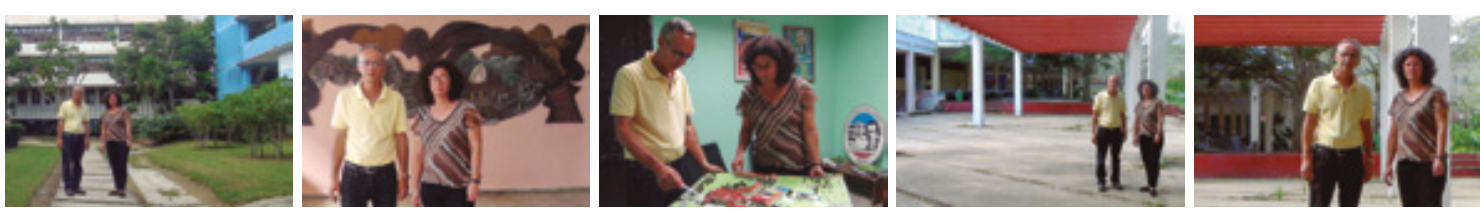

Secuencia: Recorriendo el Campus de la Universidad de Camagüey "Ignacio Agramonte Loynaz" Fotos: Omar Pino Marrero

\title{
LA ARQUITECTURA EDUCACIONAL EN CAMAGÜEY: LOS GRANDES CONJUNTOS EDUCACIONALES'
}

\author{
EDUCATIONAL ARCHITECTURE IN CAMAGÜEY:THE LARGE EDUCATIONAL \\ COMPLEXES
}

\section{Adela María García Yero², Oscar Diosdado Prieto Herrera³}

RESUMEN

\begin{abstract}
La presente reseña constituye una reflexión personal de los autores sobre tres grandes conjuntos educacionales del periodo revolucionario en Camagüey. Es parte de una caracterización en la cual se aplicó una metodología estructurada con enfoque cualitativo que permitió entender y profundizar el proceso de diseño y los factores inherentes al hecho constructivo, y con ello establecer inferencias sobre la correspondencia entre proyecto-realidad. Los resultados permitieron concluir el aporte brindado por estos conjuntos para la ciudad, con independencia de ser concebidos mediante la prefabricación, al superarse en ellos la monotonía persistente en otras instalaciones educacionales del país y alcanzar expresiones formales y funcionales de alto valor estético.
\end{abstract}

Palabras clave: centros educacionales, escuela vocacional, prefabricación, tradición.

\section{ABSTRACT}

This article presents the authors' personal reflection on three large educational complexes from the revolutionary period in Camagüey. It is part of a description that used a structured methodology with a qualitative approach to understand and delve deeper into the design process and inherent characteristics of the three educational centers, and thus establish inferences about the correspondence between projects and reality. The results indicate the contribution of these complexes to the city: although created by means of prefabrication, within and of themselves they have overcome the persistent monotony of other educational facilities around the country and have achieved a formal and functional expression of high aesthetic value.

Keywords: educational centers, vocational school, prefabrication, tradition.

Artículo recibido el 30 de agosto de 2017 y aceptado el 21 de diciembre de 2017

DOI: https://doi.org/10.22320/07196466.2017.35.052.08

[1] El artículo forma parte de los resultados parciales de la investigación que desarrollan los autores, titulada "La arquitectura de la Revolución en las provincias de Camagüey y Ciego de Ávila", Cuba.

[2] Profesora Titular del Centro de Estudios de Conservación y Desarrollo de las Construcciones de la Facultad de Construcciones,

Universidad de Camagüey "Ignacio Agramonte Loynaz". E-mail: adela.garcia@reduc.edu.cu

[3] Profesor Titular del Centro de Estudios de Conservación y Desarrollo de las Construcciones de la Facultad de Construcciones, Universidad de Camagüey "Ignacio Agramonte Loynaz". E-mail: oscar.prieto@reduc.edu.cu 


\section{INTRODUCCIÓN}

La Revolución Cubana constituyó un cambio sustancial que alcanzó todos los ámbitos de la sociedad y que tuvo un enorme apoyo popular. El joven Estado promulgó leyes, que significarían transformaciones vitales y radicales para el país, como la Campaña de Alfabetización ${ }^{4}$ y la Ley de Nacionalización de la Enseñanza de 1961, la que, con su carácter gratuito, trajo consigo un incremento de la matrícula, en especial de la enseñanza primaria.

El repertorio escolar después de la Revolución abarcó todos los niveles de enseñanza, en los contextos urbano y rural: círculos infantiles, escuelas primarias, secundarias, especializadas (tecnológicos, escuelas militares, conservatorios de música, enseñanza especial para niños con discapacidades, etc.), preuniversitarios y universidades.

Para la construcción de escuelas se contó, desde 1960, con la presencia de jóvenes arquitectos que, desde La Habana, diseñaron proyectos típicos de centros educacionales para todo el país, combinando técnicas tradicionales de construcción y el prefabricado. En este sentido, destacan, entre otros, los diseños de escuelas secundarias básicas, como las de Josefina Rebellón, para Minas y Camagüey, con el empleo de bóvedas de ladrillos y las de Mirta Merlo, ubicadas en Esmeralda, Florida y Camagüey, en las que se combinan los materiales locales con los prefabricados y la búsqueda de transparencia (Figura 1) (Soto y Morcate, 2017). 


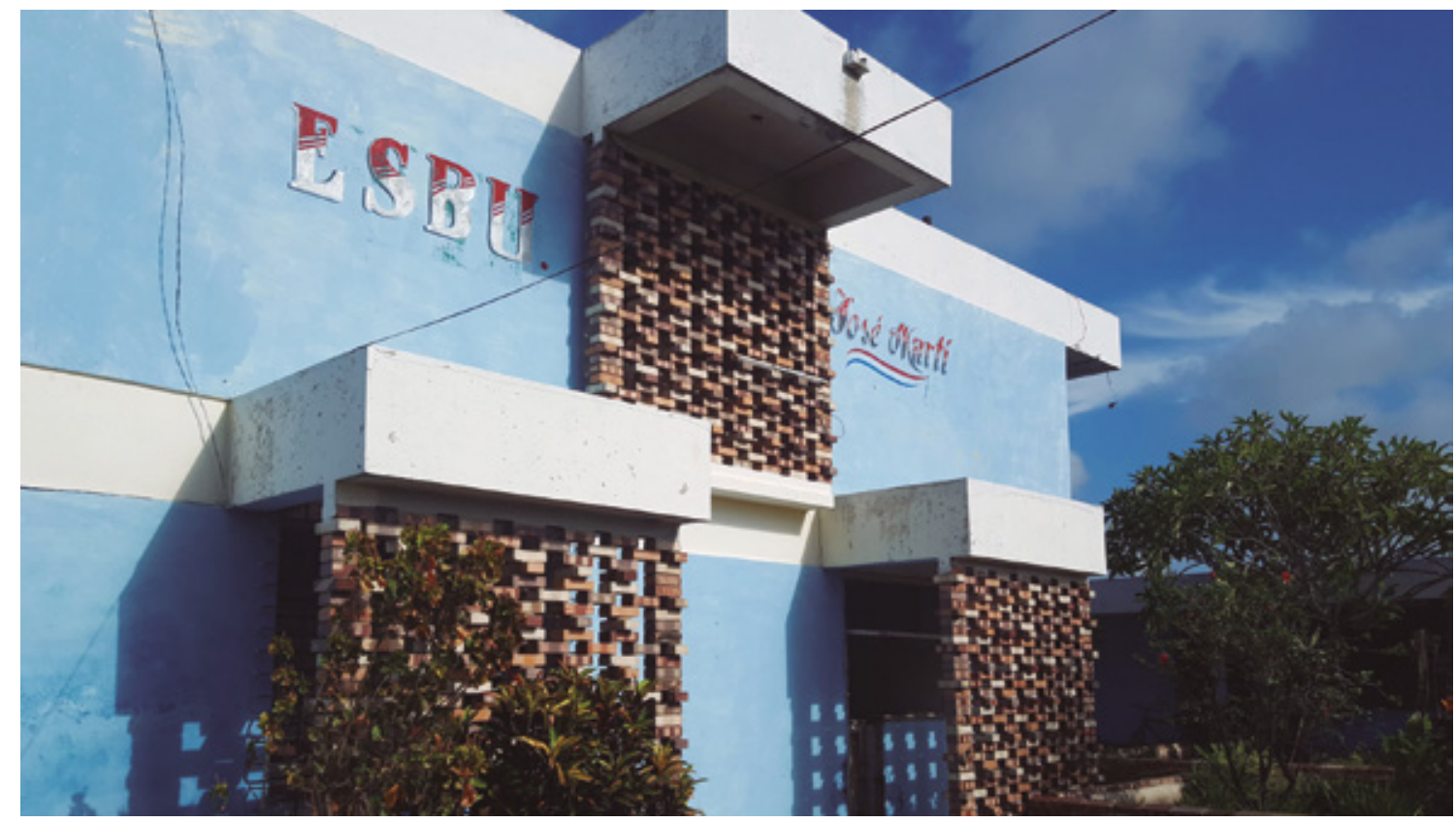

Figura 1 Escuela Secundaria Básica, municipio Florida, Camagüey, Arq. Mirta Merlo, 1961. Foto: Eider Socarrás Ferrán.

Como resultado de la difusión de estos proyectos típicos, en Camagüey se edificaron en los años 70 grandes conjuntos educacionales que, a pesar de la rigidez del sistema Girón, propia de los sistemas prefabricados, por el ingenio de los diseñadores se convierten en obras singulares, dada su adaptación contextual.

Para Camagüey, la construcción de estos centros sigue siendo un logro, ya que además del aporte para la provincia en el ámbito educacional, hoy constituyen referentes arquitectónicos de importancia nacional y local.

\section{MÉTODO}

El material se nutre de una investigación acerca de las obras de la Revolución en Camagüey, etapa poco profundizada desde la región. Se emplea una metodología cualitativa que permitió establecer los valores de estos centros educacionales. Un extenso trabajo de campo que posibilitó el estudio arquitectónico urbano concentrado en el levantamiento arquitectónico, fotográfico, morfológico del paisaje, de las técnicas y materiales constructivos. Se utilizaron los métodos de la observación participante al permitir la apreciación del fenómeno constructivo, la observación documental para la lectura de textos escritos, planos y las entrevistas estructuradas. Todo contribuyó a confrontar la realidad constructiva actual con la de proyecto, a partir de un análisis de contenido, en el cual se realizó, además, una búsqueda bibliográfica en profundidad, dada la dispersión de la información.

\section{RESULTADOS}

La ciudad de Camagüey, con más de medio milenio de fundada, se sitúa entre las más antiguas de América Latina y de Cuba. Desde el punto de vista urbanístico, prevaleció el diseño aislado que dificulta el logro de una articulación del sistema urbano. En su desarrollo, el área urbana queda enmarcada actualmente por la presencia de una vía circunvalante, mientras que su centro coincide con la ciudad tradicional o histórica, contenedora de altos valores patrimoniales y en él se encuentra el entorno declarado en el año 2008, como patrimonio cultural de la humanidad, por sus valores excepcionales. Centro heredado desde la colonia, con una imagen homogénea, reconocido históricamente como zona comercial por excelencia que coexiste con el hábitat y posee una alta densidad urbana. De este centro deriva un desarrollo radioconcéntrico, que establece un sistema de corredores radiales en el cual predominan cinco ejes de expansión, con cinturones continuos. En la zona intermedia se sitúa un alto número de viviendas, mientras en la zona externa se ubican industrias e instalaciones de servicios, entre los que se encuentran grandes centros educacionales de alcance provincial y regional, en su mayoría desarrollados después del triunfo revolucionario.

Los centros educacionales de mayor impacto comienzan con la república. Así, surge en el año 1929 el Instituto de Segunda Enseñanza (Figura 2) y durante toda la etapa republicana existió la intención de concretar una universidad, aspecto logrado solo después del triunfo la Revolución. 


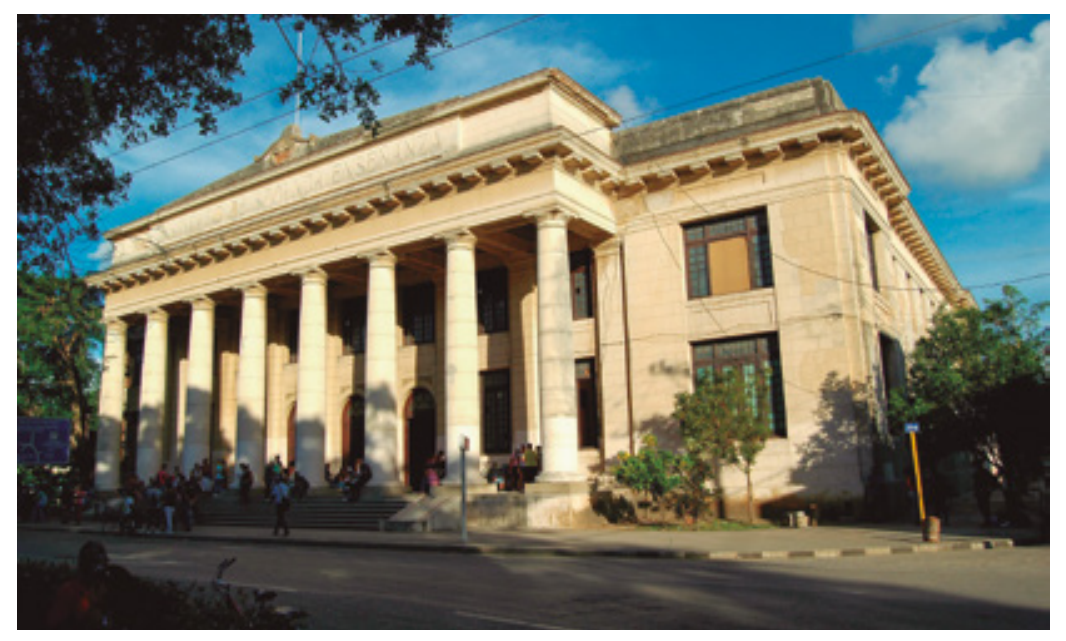

Figura 2 Instituto de Segunda Enseñanza de Camagüey, actual Instituto Preuniversitario Urbano "Álvaro Morell". Foto: Joaquín López Miranda

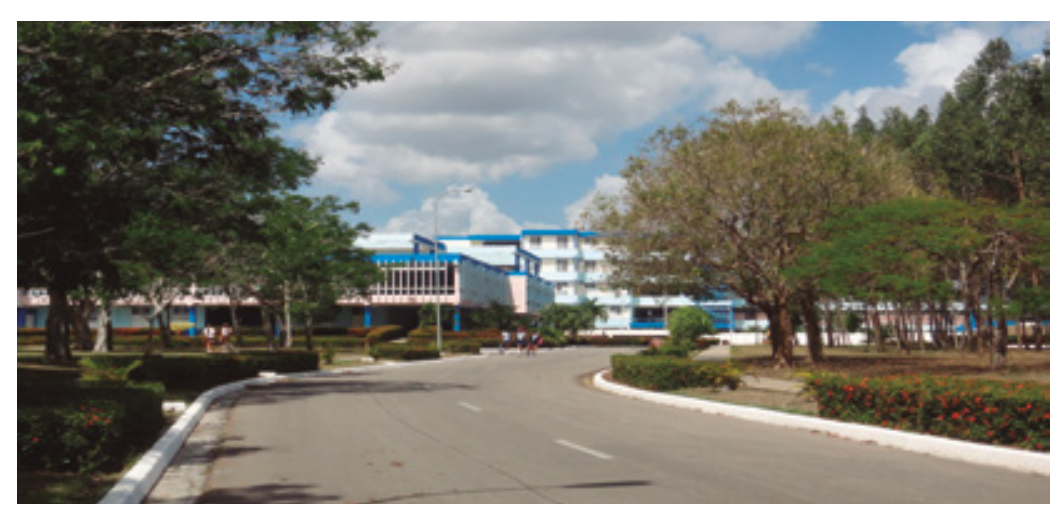

Figura 3 Escuela Vocacional Máximo Gómez Báez, de Camagüey. Foto: Archivo del CECODEC

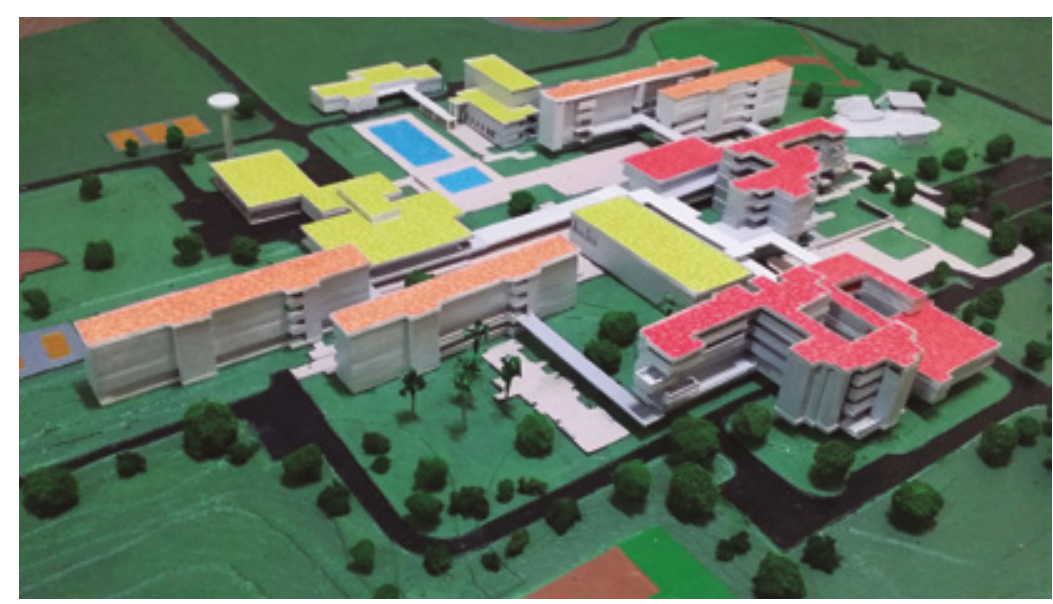

Figura 4 Maqueta de la Escuela Vocacional Máximo Gómez Báez, de Camagüey. Realizada en 1976, Reconstruida en 2017 por estudiantes de 2 do Año de la carrera de Arquitectura de la Universidad de Camagüey, con motivo del 41 aniversario de la fundación de la escuela. Foto: José Miguel Hernández Nodarse. 
En la década del setenta, se produce un proceso de institucionalización del Estado Socialista. A la par existió un incremento constructivo que abarcó el fomento de la industria, la adquisición de equipamiento, la creación de microbrigadas y de los equipos de proyectos especializados a nivel nacional en la educación, la salud y la industria. Estos equipos dedicados a las obras de la educación generaron proyectos típicos aplicables a todo el país, que se adaptaban a las características regionales. Para dar respuesta a esta urgencia provocada por los cambios en la educación cubana, se creó el sistema prefabricado Girón, a cargo de los ingenieros Aníbal Rodríguez Hoffman y Esmildo Marín y los arquitectos Josefina Rebellón Alonso, Ludy Abrahantes, Armando Galguera y José Cortiñas, quienes encabezaron nacionalmente la proyección de escuelas, incorporando dicho sistema, por su explotación intensiva, al paisaje urbano y rural del país.

En Camagüey, la ubicación de estas instalaciones escolares de gran envergadura fue concebida como parte de un gran anillo que rodea a la ciudad, conectado por la Carretera Central y las vías circunvalantes. En las inmediaciones de la Carretera Central se situó el complejo universitario y tecnológico de la salud, en conexión con los centros asistenciales de mayor importancia, y la Ciudad Escolar, antigua sede del Regimiento Ignacio Agramonte, donde se combina la enseñanza primaria y secundaria con la Dirección Provincial de Educación. Mientras que en la circunvalación se ubican la Escuela Vocacional "Máximo Gómez Báez", la Universidad de Camagüey, con sus sedes "Ignacio Agramonte Loynaz", "José Martí" y la deportiva "Manuel Fajardo"; la Escuela Vocacional de Arte "Luis Casas Romero" y las Escuelas de Iniciación Deportiva y de Perfeccionamiento Atlético "Inés Luaces". Construidas o adaptadas (como la Ciudad Escolar) por la Revolución para llevar una enseñanza de excelencia acorde a los principios del Estado cubano.

\section{Escuela Vocacional Máximo Gómez Báez}

Entre las tipologías educacionales difundidas en esta etapa se encontraron las escuelas vocacionales, donde "jóvenes arquitectos lograron doblegar la dura tecnología y diseñar un entorno poético" (Segre, 1998:17). Estaban situadas en las periferias urbanas, separadas de las principales vías de acceso por amplias franjas arborizadas, sin ser atravesadas o perturbadas por el tráfico vehicular. La Escuela Vocacional Vladimir Ilich Lenin, inaugurada en 1974 en La Habana, obra del arquitecto Andrés Garrudo, fue la primera construida y sirvió como modelo para las que se edificaron en el resto del país. Al concebir la escuela como ciudad y trabajar la escala urbana, esta obra permitió integrar los núcleos de la enseñanza secundaria con la preuniversitaria y generar un conjunto caracterizado por el empleo de patios y plazas ortogonales que destacan entre los volúmenes y el paisaje natural.
Considerada entre los mejores exponentes de estas escuelas, la vocacional Máximo Gómez Báez de Camagüey, fue terminada en 1976. Fue proyectada por el equipo liderado por el arquitecto Reinaldo Togores y compuesto por los arquitectos Heriberto Duverger como asesor; el proyectista Carlos López Quintanilla; Luis Rubio a cargo del estudio paisajístico; la estructura por los ingenieros Luis Blanco y Williams Calderón; los viales y movimiento de tierras por la ingeniera Julia Delgado; la electricidad con el ingeniero Humberto Zarraluqui y las instalaciones hidrosanitarias realizadas por el ingeniero Tony López Cruells (Figura 3).

Las premisas consideradas por Togores en su concepción se resumen de manera sintética en la expresión constructiva y funcional; la escala urbanística; la relación con la naturaleza y la caracterización simbólica (Segre: 1989: 210). Realizada por solicitud del Grupo Nacional de Construcciones Escolares, la escuela concebida para 2.500 estudiantes cubre un área de más de $45.000 \mathrm{~m}^{2}$ y se levanta como un conjunto urbano donde se aplican los criterios del Movimiento Moderno en cuanto a distribución funcional, en diálogo con la tradición local. Resulta significativa la adaptación a la topografía, el uso de los colores, el empleo de la gráfica, la continuidad espacial del conjunto y la interpenetración de los volúmenes (Figura 4).

Por medio del sistema constructivo Girón, se logró la búsqueda de una solución libre de la rigidez de los modelos desarrollados anteriormente. Para ello, se rompió:

[...] con la horizontalidad, la alineación en bloques paralelos, las culatas ciegas de los edificios y la falta de conexión en lo visual al terreno. La alineación este-oeste de los bloques docentes y la de los dormitorios estaba condicionada en buena medida por condiciones climatológicas, en particular la insolación. Pero en cambio los locales de uso común tales como biblioteca, museo, centro de cálculo, teatro, etc., se agrupan en un bloque central desarrollado en sentido norte-sur, actuando como eje de simetría en torno al cual se articula el conjunto (Togores, 2008: 4).

En el proyecto se tuvo en cuenta la restricción de la altura que imponía el desarrollo vertical en cuatro plantas impuesto por el sistema constructivo, superado a partir de las visuales logradas por el aprovechamiento del terreno y la ubicación en los extremos de los bloques docentes, limitando con el bloque central y las torres de los laboratorios escalonadas, a las cuales se les diseñaron espacios de almacenaje que permitió la configuración de elementos salientes. Se estableció el contraste con la biblioteca de dos niveles y se logró un efecto óptico de perspectiva jugado por las alturas.

Los colores se trabajaron de forma tal que los volúmenes sobresalientes de los laboratorios se pintaron de amariIlo fuerte ${ }^{5}$ para establecer la connotación volumétrica y romper con la horizontalidad del bloque central. Las culatas de los dormitorios sugieren un juego de claro- 

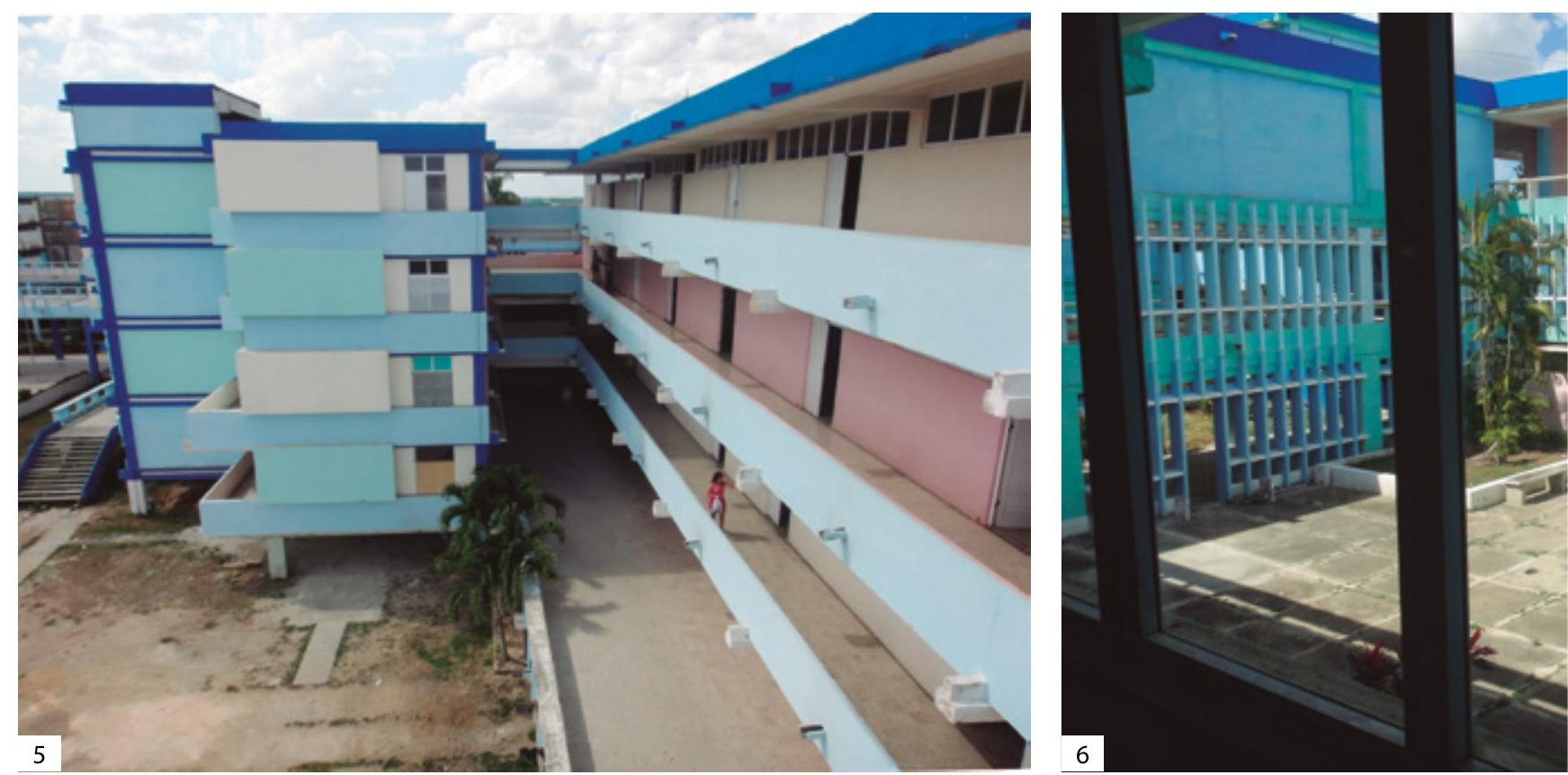

Figura 5 Culatas de edificios escuela vocacional. Foto: Archivo del CECODEC. / Figura 6 Patio interior. Universidad de Ciencias Médicas de Camagüey. Foto: Adela García Yero. / Figura 7 Estatua ecuestre de Máximo Gómez. Foto: Adela García Yero.

oscuro donde los "efectos de luz y sombra así como el uso de colores cálidos (que "avanzan" visualmente) y de colores fríos que "retroceden") dieran la impresión del relieve de que esos paredones carecen" (Ibídem, 5). Desde el punto de vista morfológico, todos los edificios que conforman el conjunto, mantienen la proporción en el planteo vertical, con un despliegue entre los dos y cuatro niveles que se adaptan a la topografía por medio de columnas que asumen los desniveles del terreno y establecen la continuidad de los recorridos y las visuales (Figura 5).

Se utilizaron las columnas altas para permitir la construcción de amplios corredores horizontales. El uso de extensos espacios abiertos hizo posible establecer cierta continuidad con la ciudad histórica y con la tradición educacional cubana: en alguna medida, son una reinterpretación del patio claustral y del patio interior de la casa colonial y republicana, espacio de intercambio, polifuncional.

Cuenta, asimismo, con la estatua ecuestre de Máximo Gómez, obra de José Delarra -que, si bien es un diseño convencional, tiene el mérito de ser la primera estatua ecuestre hecha plenamente en el país por un escultor cubano-, convertida en el elemento pregnante que distingue e identifica al plantel (Figuras 6 y 7 ).

La vocacional alcanza, con su volumetría, el tratamiento del color, los efectos ópticos de profundidad, el uso de la planta libre (actualmente ocupada en la mayoría de las zonas que lo permiten) y la creación de parques y plazas exteriores generados por el movimiento con- tinuo de la planta alrededor de los macizos de áreas verdes (Luis, 2011), una respuesta que la convierte en uno de los ejemplos de la buena arquitectura hecha por la Revolución. En resumen, esta obra constituye un exponente relevante de la aplicación del sistema Girón a nivel nacional y, a la vez, una solución moderna enlazada con la tradición y la identidad de la provincia, además de lograr articular las concepciones del Movimiento Moderno, tanto urbano como arquitectónico, con las ideas de la educación revolucionaria de la época.

\section{Universidad de Ciencias Médicas "Carlos J. Finlay", la especialización de la universidad}

La Universidad de Ciencias Médicas "Carlos J. Finlay" fue concluida en junio de 1980 . Como proyectista general, trabajó el Arq. Octavio González Morgado y estuvo a cargo la Empresa de Proyectos de Arquitectura e Ingeniería \# 11 de Camagüey. El centro universitario fue concebido para una matrícula cercana a 1.600 estudiantes de las especialidades de Medicina y Estomatología, a los que luego se añadirían otras ramas de la salud.

Las premisas de diseño impusieron la necesidad de crear un fuerte vínculo de la institución con las redes médicas existentes en el territorio, como parte de la propia concepción de la enseñanza profesional, en las cuales se relacionan conocimientos teóricos y prácticos, así como la particularidad de que los estu- 

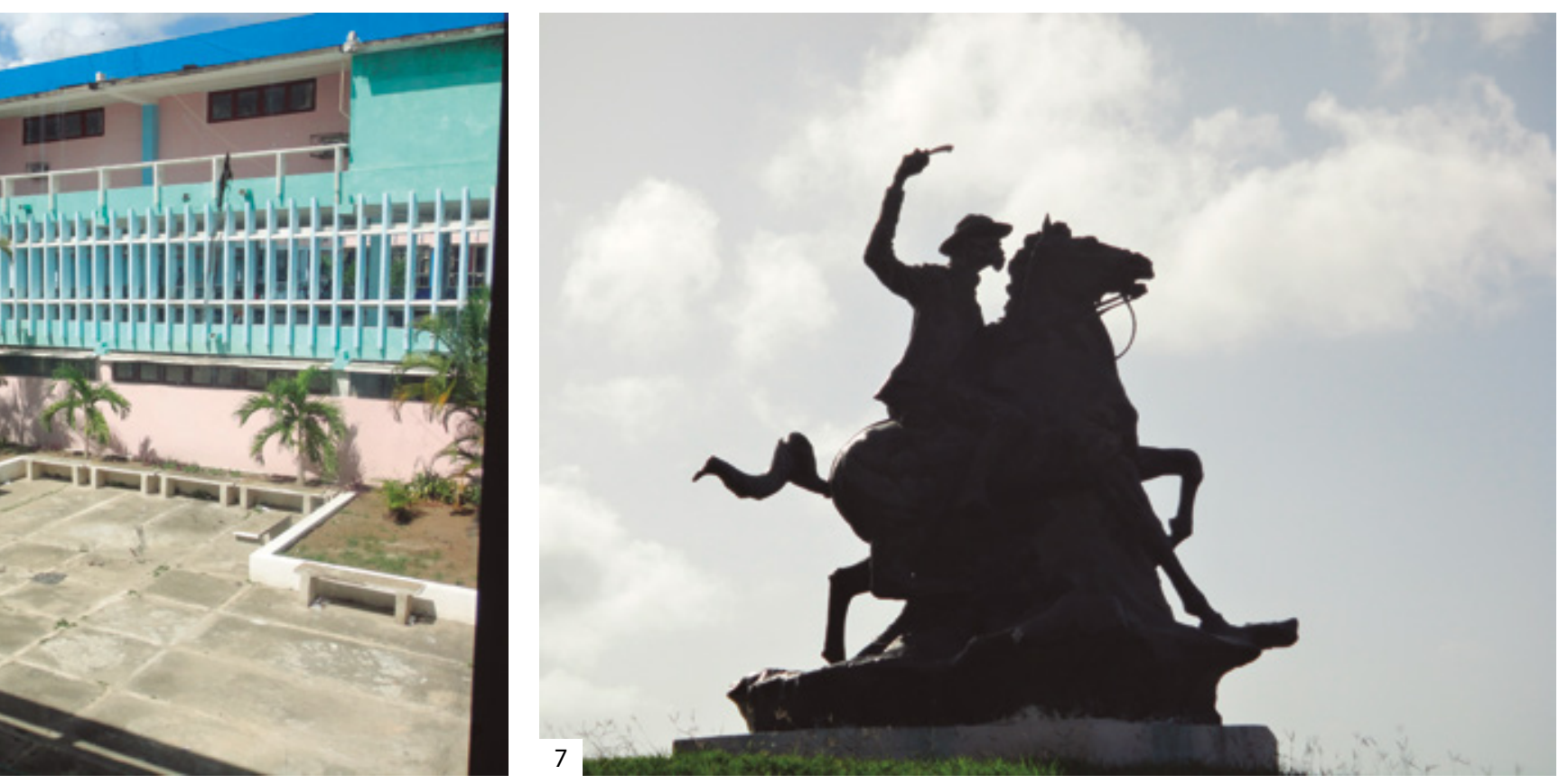

diantes se vinculan desde el tercer año directamente a los hospitales docentes. La institución universitaria funge como centro rector a nivel provincial. Esta comunicación está favorecida por su emplazamiento en la Carretera Central, principal vía de acceso de la provincia y del país, inmediato al hospital provincial clínico docente "Manuel Ascunce Domenech", cercano al hospital oncológico "María Curie", a la policlínica del reparto Previsora y con relación vial directa con el hospital provincial de Maternidad y el hospital pediátrico, así como con un amplio sistema de consultorios médicos de familia y con la ciudad. En general, agrupa un complejo muy fuerte de actividades prácticas, teóricas, investigativas y de servicios en la rama de la medicina. Es entendido, desde el proyecto, como una ciudad universitaria, por tanto, como un hecho urbano de incuestionable relevancia para la localidad.

En su planteo urbano, el río Tínima contribuye a su estructuración, al atravesar casi centralmente el espacio. Esto posibilitó concentrar las áreas: la zona docente se agrupó en la margen izquierda y en la derecha los edificios para residencia estudiantil y otros servicios. En el área académica, las edificaciones fueron ubicadas de este a oeste: docentes 1 y 2 de medicina y estomatología, donde se incluyen los laboratorios, aulas, la clínica estomatológica y el cine teatro, junto con cafetería, parqueos, plazas, patios interiores y áreas verdes. La rectoría se encuentra en la primera planta del docente de medicina. Hacia la residencia estudiantil, se sitúan los dormitorios, el comedor con el bloque de servicios y mantenimiento, bioterio, perrera y las áreas destinadas al deporte. En estas últimas, se ubica la piscina, construida para la práctica de nado sincronizado, polo acuático, baño de los estudiantes y competencias deportivas, aunque brinda también servicio a la comunidad adyacente en los meses de vacaciones; además, cuenta con un tabloncillo, conservado con buenas condiciones, que es empleado para la práctica de deportes techados como baloncesto, fútbol sala, aerobios, voleibol, entre otros. En esta zona se ubica una clínica especializada en medicina tradicional y natural que ofrece servicios a la localidad.

El proyecto no fue completado en su totalidad por las limitaciones del país, pero se logra un conjunto de fuerte expresión formal que rompe con la estricta horizontalidad del perfil urbano en el que se ubica. Logra articularse, por medio del uso del sistema Girón, propio de los grandes conjuntos educacionales del país, con la Escuela Politécnica de la Salud, obra de la arquitecta Caridad Amador, construida de forma aledaña a la residencia estudiantil y con la cual completa el complejo educacional de la salud en la ciudad.

Entre los aspectos significativos que distinguen al complejo universitario, se halla el tratamiento de la expresión formal para quebrar de ese modo la horizontalidad hacia la que tiende el conjunto, a pesar del empleo de cuatro niveles predominantes. Se buscó romper con la planimetría del entorno urbano, caracterizado por edificaciones de uno o dos niveles, a través del juego de volúmenes, con cambios de niveles y movimientos en fachadas que se apoyan en las plazas, la planta libre que se adapta a la topografía del lugar (mínimamente intervenida) y la vegetación en la 


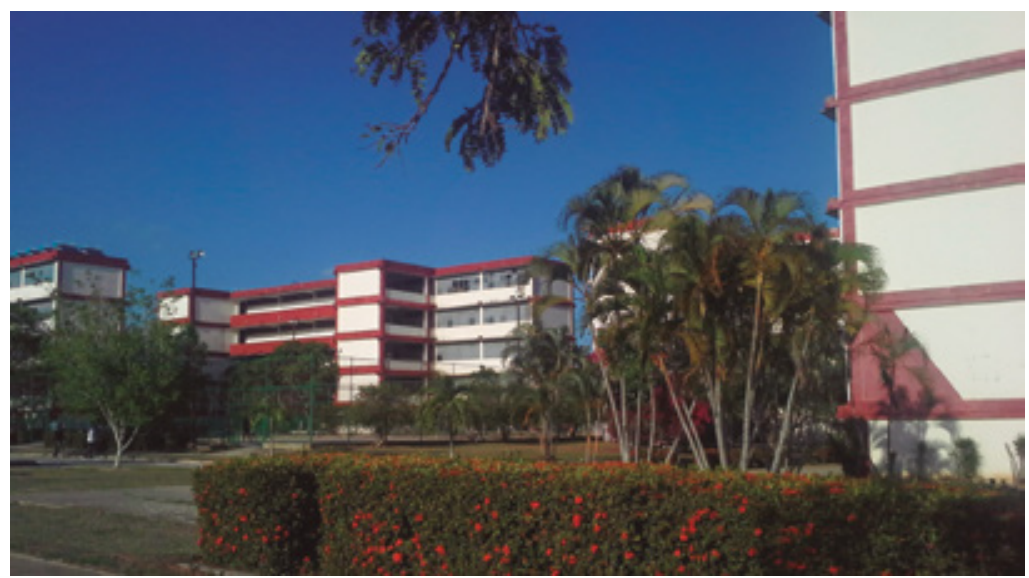

Figura 8 Bloques de edificios. Universidad de Ciencias Médicas. Foto: Adela García Yero.

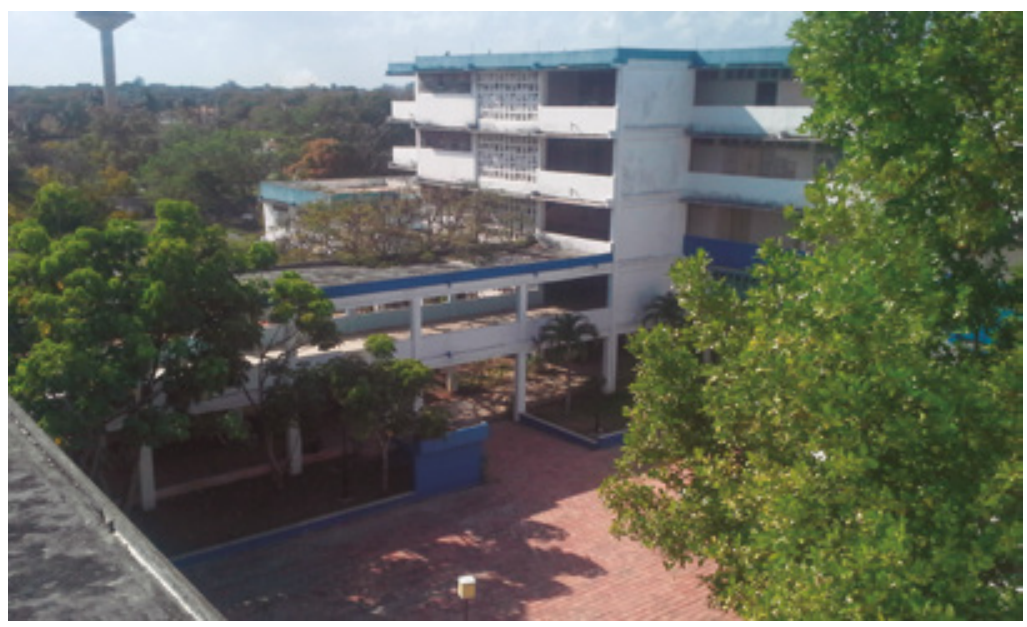

Figura 9 Detalle de los patios interiores, se privilegia el uso de los materiales locales como el barro, el tratamiento de la vegetación y del color.

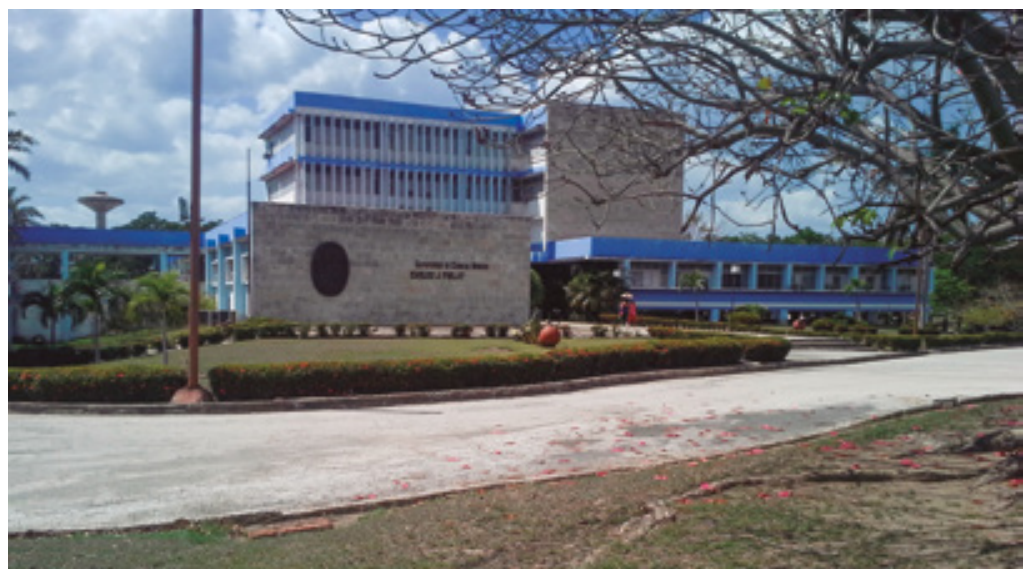

Figura 10 Acceso principal de la Universidad de Ciencias Médicas, jerarquizado por el empleo de la piedra jaimanita. Foto: Adela García Yero. 
cual destaca el estudio preciso de las áreas verdes, para crear un disfrute lúdico de espacios interiores-exteriores articulados y favorecidos por la dinámica impuesta por la organización espacial. Se integran al diseño el empleo abundante y variado de quiebrasoles, necesarios para mitigar el paso de los rayos solares y facilitar la ventilación natural y cruzada en los espacios públicos (Figura 8)

La comunicación interior se logra a través de galerías que se suceden interconectadas, con el uso de celosías, que recogen los criterios del Movimiento Moderno en torno a vincular la función con el tratamiento plástico de los elementos constructivos, a manera de enormes murales prefabricados, con motivos que varían desde la temática floral a diseños más abstractos para favorecer la transparencia exterior-interior y dar una secuencia agradable de recorridos. En ese afán de tamizar la luz para atenuar su efecto, se emplea la combinación de rejas con vitrales de colores vivos que dan pie a una estrecha vinculación del interior con los espacios exteriores y aluden a la tradición vitralista de la ciudad (Figura 9).

El proyecto potenció el uso de los materiales de terminación nacionales, donde se incluyen los locales como las losas de barro para conformar amplios espacios exteriores que disminuyen la reverberación de la luz solar y se entrelazan con la tradición alfarera de la ciudad. La piedra jaimanita, de color grisáceo y porosa textura, usada en la pared del acceso principal en oposición a la limpieza del blanco y el azul del resto de los edificios, adquiere un alto valor simbólico y estético que jerarquiza el edificio de rectoría y resalta el distintivo tratamiento de la volumetría (Figura 10).

La interpenetración de los volúmenes conjuntamente con el trabajo de los colores, en los que se prefiere el blanco ascético tratado de conjunto con el azul en el área docente, y el rojo distribuido en otros puntos, contribuyen a acentuar el tratamiento plástico espacial del conjunto urbano. La planta se concentra en la zona docente, en la que se logra una alternancia de las culatas para la ubicación de escaleras en los primeros niveles, con los balcones en azul y la presencia de grandes voladizos que se combinan con pérgolas para aumentar la dinámica del diálogo visual. En los niveles superiores, se cierran los extremos para ubicar aulas y se trabaja con el blanco y franjas azules, estableciendo el contaste de modo diferente al empleado por Togores en la vocacional.

En el conjunto se emplean volúmenes que vuelan y sobresalen, interrumpiendo la linealidad de las fachadas y se establece un movimiento brusco de las alturas a dos niveles que, a diferencia de los otros que se apoyan sobre la planta libre, nacen directamente del terreno, con lo cual se logra entonces un escalonamiento que escapa de la rigidez empleada en otras obras del sistema Girón.

\section{Universidad de Camagüey "Ignacio Agramonte Loynaz"}

La Universidad de Camagüey fue la primera fundada por la Revolución, su construcción inicia desde 1972, cumpliendo así un largo anhelo de los camagüeyanos que, desde la república abogaban por traer la enseñan- za superior a la provincia. Su alcance territorial abarca a la región centro-oriental, desde Ciego de Ávila hasta Holguín, sin excluir a estudiantes de otras zonas del país, a la vez, que brinda docencia a estudiantes extranjeros de América Latina, África, Asia y Europa.

El proyecto, con el uso del sistema Girón, concibió un conjunto de edificios articulados por galerías que se adaptan al terreno por medio de columnas que facilitan, en determinados espacios, el aprovechamiento de la planta libre. Los edificios docentes se ubicaron de este a oeste, con las aulas hacia el norte y el área de dormitorios de manera perpendicular. Se trató de aprovechar al máximo la iluminación y ventilación natural, el uso de pasillos conectores en los diferentes niveles y el desarrollo vertical en cuatro, tres y dos niveles. Las luces del sistema constructivo facilitaron el diseño de amplias aulas, talleres y laboratorios. Se aprovecha la tradición del patio para lograr zonas de parques y plazas que colaboran con el dinamismo que alcanza el conjunto, donde la incorporación de las áreas verdes, con una adecuada selección de la vegetación y el mobiliario urbano provoca interesantes juegos de perspectivas.

La concepción original estuvo a cargo de un equipo, con representación de la Empresa de Proyectos de Obras Integradas \# 4, de La Habana y de la Empresa de Proyectos de Ingeniería y Arquitectura \# 11 de Camagüey, conjuntamente con inversionistas de la propia universidad. Por la primera, y como proyectista principal, estuvo la arquitecta Oria Mansito y, como proyectistas, los arquitectos Josefina Montalván y José Fernández; de la empresa provincial, los ingenieros estructurales Francisco Monroy y Heriberto Sardiñas y el arquitecto Rafael Rivero, mientras que por la inversión, estuvo el ingeniero estructural y profesor de la universidad Ernesto Pérez Cerezalez, e indistintamente participaron proyectistas como el arquitecto Oscar Prieto y Rafael Rodríguez, que diseñaron el Paraninfo; el ingeniero Miguel Ávalos y la arquitecta Amada Zamora (Figura 11).

El conjunto comenzó como escuela de formación pedagógica, con los edificios donde hoy se ubican la Facultad de Ciencias Agropecuarias como bloque socio-administrativo, los dormitorios, el comedor viejo y los laboratorios de Ciencias Básicas. En el año 1975, se concreta como universidad y los primeros objetos de obras concebidos fueron el Centro de Información y el edificio de Ingeniería (actual Facultad de Electromecánica); luego, en 1979, se le agrega la Facultad de Construcciones y, gradualmente, se va configurando el conjunto (Figura 12). Los últimos edificios terminados fueron los correspondientes a la residencia estudiantil de extranjeros (Pérez, 2017) (Figura 13).

Si bien se concibió como un proyecto integrador, la realidad constructiva distó de la idea, al ejecutarse en largas etapas y estar sometido a constantes transformaciones, lo que atenta contra la integración del conjunto: prima, en definitiva, la fragmentación. Sin embargo, constituye una obra a considerar por la constante adecuación constructiva y las nuevas soluciones a las posibilidades y necesidades de la educación superior del país. 


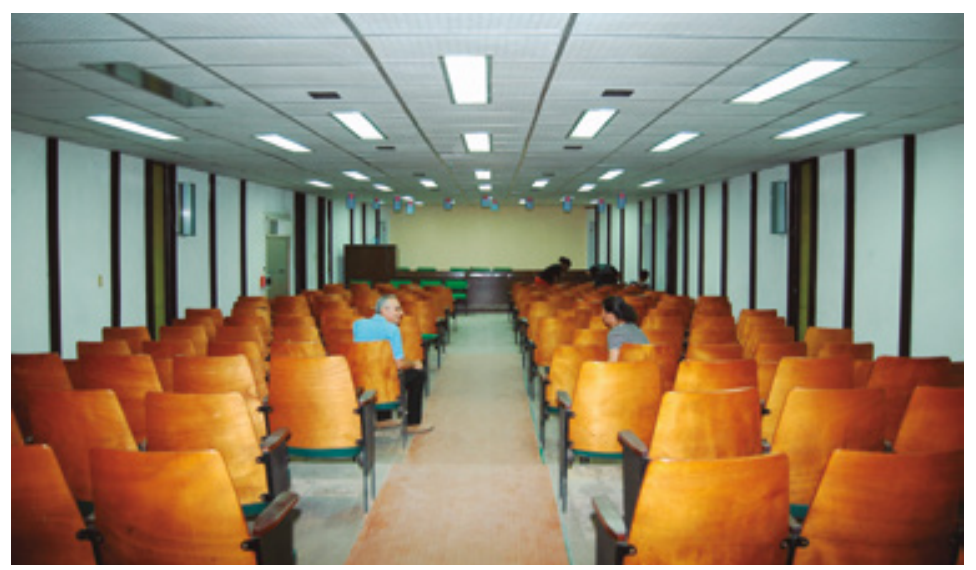

Figura 11 Paraninfo. Foto: Arq. Joaquín López Miranda.

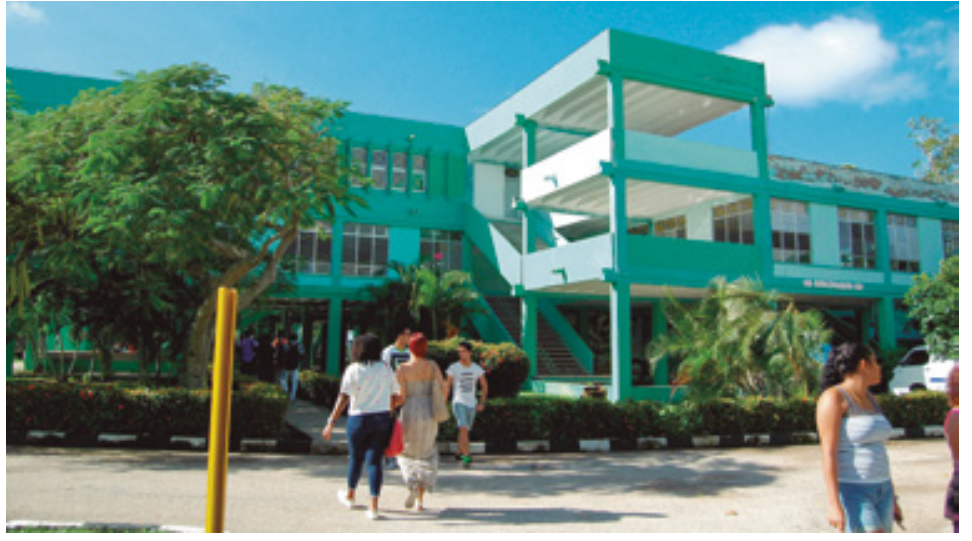

Figura 12 Comedor Universidad. Foto: Arq. Joaquín López Miranda.

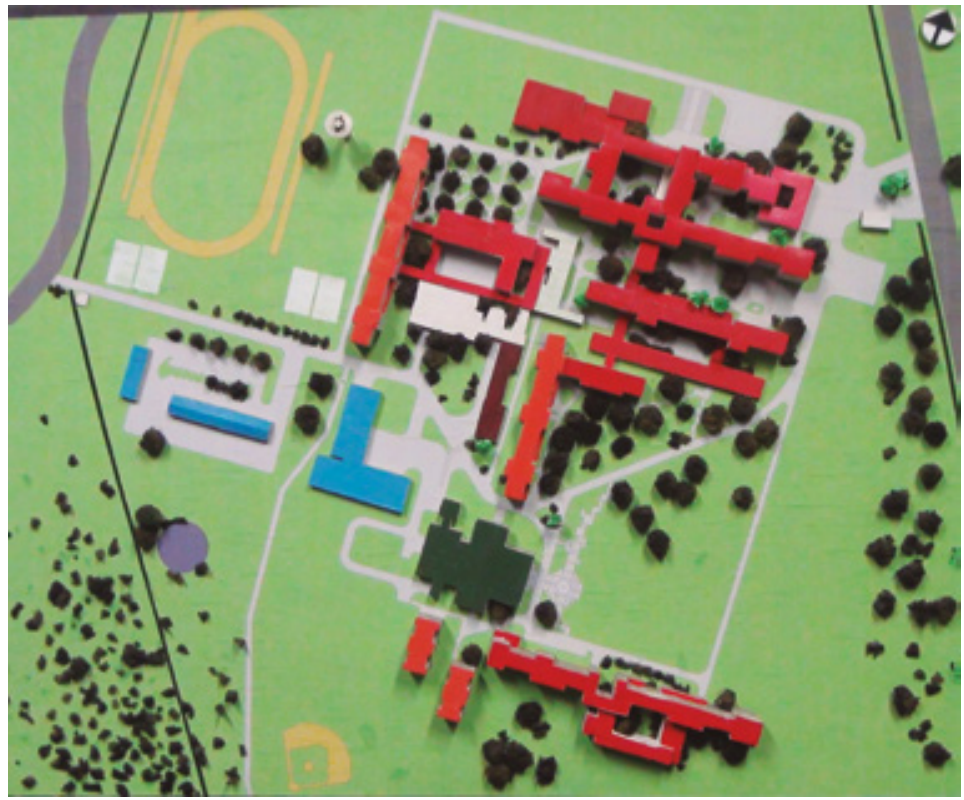

Figura 13 Maqueta Universidad de Camagüey. Foto: Omar Pino Marrero 


\section{CONCLUSIONES}

Camagüey, como ciudad en desarrollo, debe a la Revolución la creación de tres grandes centros escolares que posibilitó un despegue en la formación académica de la provincia. Estos centros fueron desarrollados mediante el sistema prefabricado Girón en combinación con el empleo de materiales locales y la asimilación de concepciones espacio-ambientales heredadas de la arquitectura precedente.

La inclusión de plazas y parques distinguen estas realizaciones que se entrelazan con la tradición hispana del patio claustral, la articulación por medio de galerías, el diseño de espacios verdes y el respeto a la topografía; todas premisas de diseño empleadas en estos tres conjuntos.

Es importante resaltar cómo esta arquitectura, a pesar de la rigidez impuesta por los sistemas prefabricados, posibilitó alcanzar una expresión propia que la distingue y singulariza en el país.

La vocacional "Máximo Gómez Báez" de Camagüey se encuentra en la lista de obras protegidas por el DOCOMOMO y se convierte en un referente singular, de gran relevancia a nivel nacional, al unificar morfológicamente el conjunto por medio de la proporción de los edificios, manejar de forma excelente la diferencia de alturas y lograr la articulación al contexto. El manejo de los volúmenes y los colores se considera otro aporte, ya que ambos propician efectos ópticos de profundidad que contribuyen a reforzar el carácter simbólico espacial que dialoga con la tradición local.

Esta arquitectura es manifestación de la cultura de su tiempo, aquí se intenta sobrepasar los límites impuestos por la emergencia, la tipificación y la prefabricación, en la búsqueda de una respuesta identitaria capaz de adaptarse al ambiente natural y construido, para trascender por su profundo sentido humanista.

\section{REFERENCIAS BIBLIOGRÁFICAS}

LUIS RODRÍGUEZ, Eduardo (ed.). La arquitectura el Movimiento Moderno. Selección de obras del Registro Nacional, La Habana: Ed. Unión, 2011.

PÉREZ CEREZALEZ, Ernesto. Entrevista realizada por Adela María García Yero (2017).

SEGRE, Roberto. Arquitectura y urbanismo de la Revolución cubana. La Habana: Ed. Pueblo y Educación, 1989.

SEGRE, Roberto. Encrucijadas de la arquitectura en Cuba: realismo mágico, realismo socialista y realismo crítico. Conferencia pronunciada en el ciclo «La Habana 1898-1998», organizada por la Fundación Cultural COAM del Colegio Oficial de Arquitectos de Madrid, España, 1998.

SOTO, Milene y MORCATE, Flora. Arquitectura educacional 1959-2017. Cuba. Ponencia presentada al Encuentro Académico Cubano-Alemán, Universidad de Kassel, Alemania, marzo-abril 2017.

TOGORES, Reinaldo. Entrevista realizada por Meylis Cruz Fernández, 2008 [en línea]. [Consultado octubre de 2016]. Disponible en: http://www.togores. net/arquitectura. 